Notre Dame Journal of Formal Logic

Volume 22, Number 4, October 1981

\title{
A Property Which Guarantees Termination in Weak Combinatory Logic and Subtree Replacement Systems
}

ALBERTO PETTOROSSI*

1 Introduction It is well-known that recursive equations may be conveniently used for defining functions and specifying their computations. A particular syntactic system for writing such equations is Weak Combinatory Logic. Such logical formalism, even if not particularly appealing to computer scientists because of its syntactical characteristics, could be useful when we want to consider also untyped functions and would like to avoid the extra problems due to the presence of variables and their bindings, as in type-free $\lambda$-calculus [1].

Those equations may be considered as production rules for deriving "simpler" terms from more complex ones, and in that case the crucial problem of the existence of the "normal form" arises: does there exist the "simplest" derivable term? Is it unique?

In this paper we study that problem and give the definition of a property which is sufficient, under some hypotheses, for assuring the existence of such

\footnotetext{
*This work was supported by the Consiglio Nazionale della Ricerche. I would like to thank Professor A. Robinson and Professor L. Sanchis for their support and encouragement in studying combinatory logic during my visit to Syracuse University. They and all the people at the Computer and Information Science Department made my studying very enjoyable and fruitful. I learned a great deal from their enthusiastic and stimulating teaching. Many thanks also to Dr. G. Ausiello at CSSCCA-CNR, Dr. S. Micali, Professor C. Böhm at the Istituto Mathematico in Rome, and especially Dr. M. Venturini Zilli at IAC-CNR and the anonymous referee for suggestions and criticisms.
} 
normal forms, extending previous known results [2]. Also included is the case in which a term may be obtained, during the rewriting process, via duplication of subterms. The result is also applicable to subtree replacement systems and therefore to all computational processes for which such systems may provide useful semantical models.

2 Initial definitions and notations In order to avoid ambiguity we first recall the definition of Weak Combinatory Logic (WCL) [2]. The alphabet is:

$$
\begin{array}{ll}
I, K, S & \text { constants (or basic combinators) } \\
(,) & \text { special symbols for building terms } \\
>,=, \geqslant & \text { binary infixes for building formulas } \\
x, y, \ldots, x_{1}, x_{2}, \ldots & \text { variables. }
\end{array}
$$

Terms are defined as follows:

i. a constant or a variable is an atomic term

ii. an applicative combination of two terms $\alpha_{1}$ and $\alpha_{2}$, denoted by $\left(\alpha_{1} \alpha_{2}\right)$, is a term.

We generally assume left associativity, so that $\alpha_{1} \alpha_{2} \ldots \alpha_{n}$ stands for $\left(\ldots\left(\alpha_{1} \alpha_{2}\right) \ldots \alpha_{n}\right)$.

Combinators are terms without variables.

The reduction axioms and inference rules of WCL are:

$$
\text { 1. } I x_{1}>x_{1}
$$

2. $K x_{1} x_{2}>x_{1}$

3. $S x_{1} x_{2} x_{3}>x_{1} x_{3}\left(x_{2} x_{3}\right)$ (reduction axiom of $I$ ) (reduction axiom of $K$ )

4. If $\alpha_{1}>\alpha_{2}$ then $\alpha_{1}=\alpha_{2}$

5. Reflexivity holds for $>$

$6 . \geqslant$ is the reflexive transitive closure of $>$

7. Reflexivity, symmetry, and transitivity hold for $=$

8. a. If $\alpha_{1}>\alpha_{2}$ then $\alpha_{0} \alpha_{1}>\alpha_{0} \alpha_{2}$ b. If $\alpha_{1}>\alpha_{2}$ then $\alpha_{1} \alpha_{0}>\alpha_{2} \alpha_{0}$.

To simplify some of the following definitions we write the reduction axioms using variables (e.g., $x_{i}$ ) instead of generic terms (e.g., $\alpha_{i}$ ). $\equiv$ denotes syntactical identity. We write $\alpha>_{n} \beta$ for showing that $\beta$ is obtained from $\alpha$ by $n$ applications of reduction axioms.

We can introduce in WCL some other constants (or basic combinators) defining them either in terms of $I, K$, and $S$ or by giving a reduction axiom. This is a consequence of the Combinatory Completeness Theorem [2]. For example we can introduce a constant $B$ by giving either $B \equiv S(K S) K$ or $B x_{1} x_{2} x_{3}>x_{1}\left(x_{2} x_{3}\right)$.

Let us now introduce some other definitions. A subbase $\mathcal{B}$ is a nonempty (possibly infinite) set of basic combinators $\mathcal{B}=\left\{X_{1}, \ldots, X_{n}\right\}$. The applicative closure $\mathcal{B}^{+}$of a subbase $\beta$ is the set of all finite applicative combinations of basic combinators in $\mathcal{B}$. As far as notations are concerned,

$a, \beta, \ldots \quad$ denote subbases

$X, Y, \ldots, K, S, \ldots$ denote basic combinators 

$x, y, \ldots \quad$ denote variables
$\alpha, \beta, \ldots \quad$ denote terms
$\chi, \phi, \psi, \ldots \quad$ denote combinators.

We also use subscripts and superscripts, if necessary. Let us suppose that we are given a subbase $\beta$ and a term $\alpha$ built out of basic combinators in $\beta$ and variables. The set $S_{\alpha}$ of subterms of $\alpha$ is defined as follows:

i. if $\alpha$ is a basic combinator or a variable (atomic subterm) then $S_{\alpha}=\{\alpha\}$

ii. if $\alpha \equiv\left(\alpha_{1} \alpha_{2}\right)$ then $S_{\alpha}=S_{\alpha_{1}} \cup S_{\alpha_{2}} \cup\{\alpha\}$.

The set of proper subterms of $\alpha$ is $S_{\alpha}-\{\alpha\}$. follows:

The set $T_{\alpha}$ of right applied subterms (or branches) of $\alpha$ is defined as

i. if $\alpha$ is a basic combinator or a variable then $T_{\alpha}=\{\alpha\}$

ii. if $\alpha \equiv\left(\alpha_{1} \alpha_{2}\right)$ then $T_{\alpha}=T_{\alpha_{1}} \cup\left\{\alpha_{2}\right\}$.

We say that the basic combinator $X$ with reduction axiom $X x_{1} \ldots x_{m}>\beta$ is a proper combinator iff $\beta$ is an applicative combination of variables in the set $\left\{x_{1}, \ldots, x_{m}\right\}$. We also say that $X$ has duplicative effect iff $\exists x_{i}, 1 \leqslant i \leqslant m$, such that $x_{i}$ occurs in $\beta$ more than once; $X$ has compositive effect iff a right applied subterm of $\beta$ is not a variable.

Example 1:

i. $W$ such that $W x_{1} x_{2}>x_{1} x_{2} x_{2}$ is a proper combinator with duplicative effect.

ii. $B$ such that $B x_{1} x_{2} x_{3}>x_{1}\left(x_{2} x_{3}\right)$ is a proper combinator with compositive effect.

iii. Given $\mathcal{B}=\{S, K\}$ and $\alpha \equiv S(K S) x_{2}$,

$$
S_{\alpha}=\left\{S, K S, K, x_{2}, S(K S), S(K S) x_{2}\right\} \text { and } T_{\alpha}=\left\{S, K S, x_{2}\right\} .
$$

Given a term $\alpha$ we define the corresponding marked term marked $(\alpha)$ as follows:

$$
\operatorname{marked}(\alpha)=\text { marked } 1(\langle\alpha, 0\rangle)
$$

where

$$
\begin{aligned}
\text { marked } 1(\langle\alpha, n\rangle)= & \langle\alpha, n\rangle \text { if } \alpha \text { is a basic combinator or a variable } \\
= & \left(\text { marked } 1\left(\left\langle\alpha_{1}, n+1\right\rangle\right), \text { marked } 1\left(\left\langle\alpha_{2}, n\right\rangle\right)\right) \\
& \text { if } \alpha \equiv\left(\alpha_{1} \alpha_{2}\right) .
\end{aligned}
$$

Example 2:

i. $\operatorname{marked}\left(S x_{1} x_{2} x_{3}\right)=\left(\left(\left(\langle S, 3\rangle,\left\langle x_{1}, 2\right\rangle\right),\left\langle x_{2}, 1\right\rangle\right),\left\langle x_{3}, 0\right\rangle\right)$

ii. marked $\left(x_{1} x_{3}\left(x_{2} x_{3}\right)\right)=\left(\left(\left\langle x_{1}, 2\right\rangle,\left\langle x_{3}, 1\right\rangle\right),\left(\left\langle x_{2}, 1\right\rangle,\left\langle x_{3}, 0\right\rangle\right)\right)$.

Therefore a marked term is a term in which all basic combinators and variables are associated with an integer. If we represent a term as a binary tree according to its applicative structure, then that integer is the number of "left choices" one has to make for going from the root of the tree to the considered basic combinator or variable. 
Example 3: Using a binary tree representation,

$\operatorname{marked}\left(x_{1} x_{3}\left(x_{2} x_{3}\right)\right)=$

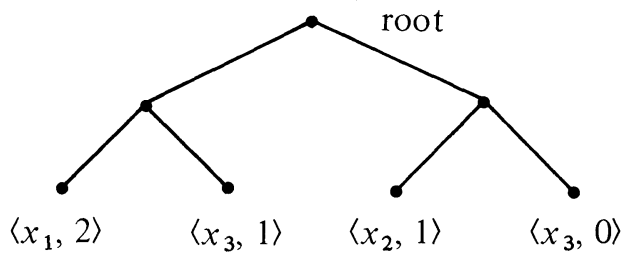

\section{The nonascending property and the basic theorem}

Definition 1 We say that a proper combinator $X$ with reduction axiom $X x_{1} x_{2} \ldots x_{m}>\beta$ has nonascending property (NA property) iff $\forall i$, for $1 \leqslant i \leqslant m$, if $\left\langle x_{i}, p\right\rangle$ occurs in $\operatorname{marked}\left(X x_{1} \ldots x_{m}\right)$ and $\left\langle x_{i}, q\right\rangle$ occurs in $\operatorname{marked}(\beta)$ then $p \geqslant q$.

Example 4:

i. $X$ such that $X x_{1} x_{2} x_{3}>x_{1} x_{2} x_{2}$ has NA property, because

marked $\left(X x_{1} x_{2} x_{3}\right)=\left(\left(\left(\langle X, 3\rangle,\left\langle x_{1}, 2\right\rangle\right),\left\langle x_{2}, 1\right\rangle\right),\left\langle x_{3}, 0\right\rangle\right)$ and $\operatorname{marked}\left(x_{1} x_{2} x_{2}\right)=\left(\left(\left\langle x_{1}, 2\right\rangle,\left\langle x_{2}, 1\right\rangle\right),\left\langle x_{2}, 0\right\rangle\right)$.

ii. $S$ such that $S x_{1} x_{2} x_{3}>x_{1} x_{3}\left(x_{2} x_{3}\right)$ does not have NA property, because

$\left\langle x_{3}, 0\right\rangle$ occurs in marked $\left(S x_{1} x_{2} x_{3}\right)$ and

$\left\langle x_{3}, 1\right\rangle$ occurs in marked $\left(x_{1} x_{3}\left(x_{2} x_{3}\right)\right)$.

For the usual concepts of "normal form", "redex", and "reductum" in WCL, see [2].

Theorem 1 (Basic Theorem) Given a proper combinator $X$ with NA property and without compositive effect $\forall \chi \in\{X\}^{+} \chi$ has normal form.

In order to prove Theorem 1 we first introduce more definitions and prove some lemmas.

Definition 2 Given a term $\alpha$ we define the set $R_{\alpha}$ of terms reachable from $\alpha$ as follows: $R_{\alpha}=\{\beta \mid \alpha \geqslant \beta\}$.

Definition 3 Given a term $\alpha$ and a pair $\langle X, n\rangle$ (where $X$ is a basic combinator) which occurs in marked $(\alpha), n$ is the copy-number (or c-number) of that occurrence of $X$ in $\alpha$. We also say that such an occurrence of $X$ is associated with the c-number $n$ or, simply, $X$ is with c-number $n$ or $X$ has c-number $n$.

Definition 4 We say that a combinator $\chi$ cycles iff $\chi>_{k} \chi$ for some $k \geqslant 1$.

Remark 1: In proving the following lemmas and Theorem 1 we consider all reductions to be leftmost outermost. This is a safe strategy for obtaining the normal form (see [2]).

Remark 2: From now on, unless otherwise stated, we will consider that the basic proper combinator $X$ has NA property and does not have compositive effect. 
Remark 3: In what follows we say that "the maximum c-number in $\chi \in\{X\}^{+}$is $m$ " or " $\chi$ has maximum c-number $m$ " as abbreviations for: " $m$ is the maximum integer such that $\langle X, m\rangle$ occurs in $\operatorname{marked}(\chi)$ ".

Lemma $1 \quad \forall \chi \in\{X\}^{+} R_{\chi}$ is finite.

Proof: Let $m$ be the maximum c-number in $\chi$. Since $X$ has NA property and does not have compositive effect, $\forall \phi \in R_{\chi}$ such that $\chi \geqslant \phi, \phi$ must be an applicative combination of at most $m$ subterms of $\chi$, otherwise at least one $X$ in $\phi$ would have a c-number greater than $m$. Since the set of all subterms of $\chi$ is finite, $R_{\chi}$ is finite.

Lemma 2 If $\chi \in\{X\}^{+}$does not have normal form then there exists $\bar{\chi} \in\{X\}$ such that $\bar{\chi} \equiv X \phi_{2} \ldots \phi_{n}$, where $\forall \phi_{i}$, for $2 \leqslant i \leqslant n, \phi_{i}$ is in normal form, and $\bar{\chi}$ has no normal form.

Proof: Let $\Omega$ be the set of all terms in $\{X\}^{+}$without normal form. Suppose $\Omega \neq \phi$. We can order $\Omega$ using the "proper subterm" relation. It is a well ordering and therefore there exists $\bar{\chi} \epsilon \Omega$ whose proper subterms have normal form.

Definition $5 \quad$ A reduction $\alpha>_{1} \beta$ is called a head reduction when either $\alpha \equiv \rho$ or $\alpha \equiv \rho \alpha_{2} \ldots \alpha_{n}$ with $n \geqslant 2$ where $\rho$ is the reduced redex.

Lemma 3 Given $\chi_{0} \in\{X\}^{+}$such that $\chi_{0} \equiv X \phi_{2} \ldots \phi_{m}$ where all $\phi_{i}$ 's with $2 \leqslant i \leqslant n$ are in normal form, if $\chi_{0}>_{1} \chi_{1}>_{1} \ldots>_{1} \chi_{i}>_{1} \ldots$ then $\forall i \geqslant 0$, $\chi_{i}>_{1} \chi_{i+1}$ is a head reduction.

Proof: Immediate because $X$ does not have compositive effect.

Definition 6 Given the head reduction $\alpha>_{1} \beta$, that is $\rho \alpha_{2} \ldots \alpha_{n}>_{1}$ $\rho^{\prime} \alpha_{2} \ldots \alpha_{n}$ where $\rho$ is the redex and $\rho^{\prime}$ is the contractum, we say that a subterm $\sigma_{\alpha}$ of $\alpha$ immediately produces the subterm $\sigma_{\beta}$ of $\beta$ (or $\beta$ immediately derives from $\alpha$ ) iff either $\exists i 2 \leqslant i \leqslant n$ such that $\sigma_{\alpha} \equiv \sigma_{\beta} \equiv \alpha_{i}$ or $\sigma_{\alpha}$ and $\sigma_{\beta}$ are subterms of $\rho$ and $\rho^{\prime}$ respectively and both correspond to the same variable $x_{i}$ of the reduction axiom applied in the given reduction. If $\sigma_{\alpha}^{(1)} \sigma_{\alpha}^{(2)}$ immediately produces $\sigma_{\beta}^{(1)} \sigma_{\beta}^{(2)}$ we also say that $\sigma_{\alpha}^{(i)}$ immediately produces $\sigma_{\beta}^{(i)}$ (or $\sigma_{\beta}^{(i)}$ immediately derives from $\left.\sigma_{\alpha}^{(i)}\right)$ for $i=1,2$.

Example 5: In
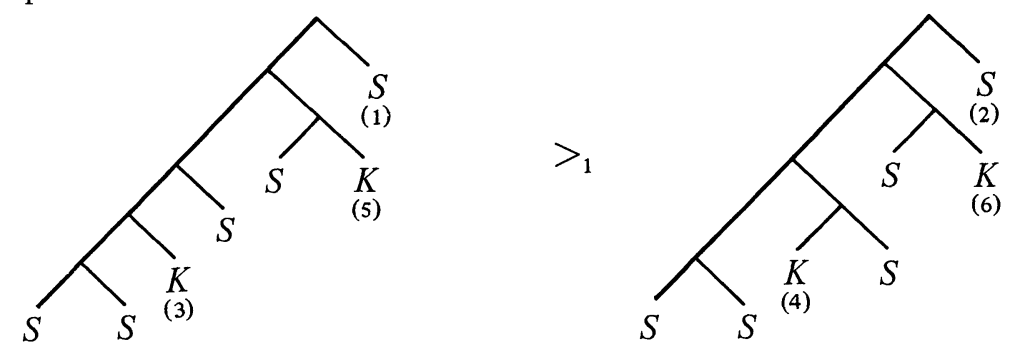

which is a head reduction, $\underset{(1)}{S}$ immediately produces $\underset{(2)}{S}$ (because they both correspond to $\alpha_{3}$, writing the reduction as $\left.\rho \alpha_{2} \alpha_{3}>_{1} \rho^{\prime} \alpha_{2} \alpha_{3}\right) ; K$ immediately derives from $K$ (because they both correspond to the variable $x_{2}$ of the axiom 
$S x_{1} x_{2} x_{3}>x_{1} x_{3}\left(x_{2} x_{3}\right)$ ); and $K$ immediately produces $K$ (because $S K$ immedi(5)

ately produces $S \underset{(6)}{K}$ since they both correspond to $\alpha_{2}$ ).

Definition 7 Given a sequence of head reductions $\alpha \equiv \alpha_{0}>_{1} \ldots>_{1} \alpha_{n} \equiv \beta$, we say that a subterm $\sigma_{\alpha}$ of $\alpha$ produces a subterm $\sigma_{\beta}$ of $\beta$ (or $\sigma_{\beta}$ derives from $\sigma_{\alpha}$ ) iff there exists a sequence of subterms $\sigma_{\alpha_{0}}$ of $\alpha_{0}, \sigma_{\alpha_{1}}$ of $\alpha_{1}, \ldots, \sigma_{\alpha_{n}}$ of $\alpha_{n}$ such that $\forall k, 1 \leqslant k \leqslant n, \sigma_{\alpha_{k-1}}$ immediately produces $\sigma_{\alpha_{k}}$.

Example 6: Given $\alpha_{0} \equiv K K K(S S K) K K>_{1}, \alpha_{1} \equiv K(S S K) K K>_{1}, \alpha_{2} \equiv S S K K>_{1}$,

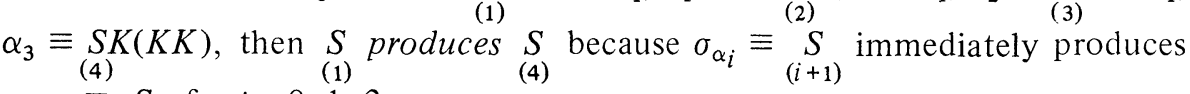
$\sigma_{\alpha_{i+1}} \equiv \underset{(i+2)}{S}$ for $i=0,1,2$.

Remark 4: If $\sigma_{\alpha}$ produces $\sigma_{\beta}$ then $\sigma_{\alpha} \equiv \sigma_{\beta}$.

Lemma 4 Given $\chi_{1}, \chi_{2} \in\{X\}^{+} \chi_{1}>_{k} \chi_{2}$ with $k \geqslant 1$. Let us suppose that $\chi_{1}$ and $\chi_{2}$ have the same maximum c-number $m$ : (i) $A$ copy of $X$ with c-number $m$ in $\chi_{1}$ produces 0 or 1 copy of $X$ with c-number $m$ in $\chi_{2}$. (ii) $A$ copy of $X$ with $c$-number $m$ in $\chi_{2}$ can derive only from a copy of $X$ in $\chi_{1}$ with c-number $m$.

Proof: (i) Given $X x_{1} \ldots x_{m}>\beta$, since $X$ does not have compositive effect, the branches of $\beta$ are variables, i.e., elements of the set $\left\{x_{1}, \ldots, x_{m}\right\}$. Since $X$ has NA property at most one copy of $x_{i}$, which has c-number $m-i$ in $X x_{1} \ldots x_{m}$, has the same c-number $m-i$ in $\beta$. (ii) Obvious, because $X$ has NA property.

Lemma 5 Given $\chi_{0} \in\{X\}^{+}$if $\chi_{0}>_{1} \chi_{1}>_{1} \ldots>_{1} \chi_{k} \equiv \chi_{0}$ then $\forall i, j, 0 \leqslant i$, $j \leqslant k$, the maximum c-number in $\chi_{i}$ is equal to the maximum c-number in $\chi_{j}$.

Proof: Since $X$ has NA property, the maximum c-number cannot increase. If it decreases, $\chi$ cannot cycle.

Lemma 6 Given $\chi_{0} \epsilon\{X\}^{+}$if $\chi_{0}>_{1} \chi_{1}>_{1} \ldots>_{1} \chi_{k} \equiv \chi_{0}$ then $\forall i, j, 0 \leqslant i$, $j \leqslant k$, the number of copies of $X^{\prime}$ 's in $\chi_{i}$ with maximum c-number is equal to the number of copies of $X$ 's in $\chi_{j}$ with maximum c-number.

Proof: The maximum c-number in $\chi_{i}$ is equal to the maximum c-number in $\chi_{j}$ by Lemma 5 . Since $X$ has NA property, the number of copies of $X$ 's with maximum c-number in $\chi_{i}$ cannot increase in $\chi_{j}$ for $j>i$ by Lemma 4 . If it decreases then $\chi_{0}$ cannot cycle.

Lemma 7 Given $X \phi_{1} \ldots \phi_{k}>_{1} \beta$ where $\phi_{i} \in\{X\}^{+}$with $i=1, \ldots, k$, if $\phi_{i}$ has maximum c-number $m_{i}$ in $\left(X \phi_{1} \ldots \phi_{k}\right)$ for $i=1, \ldots, k$ then the maximum $c$-number in $\beta$ is at most $\max \left(m_{1}, \ldots, m_{k}\right)$.

Proof: Immediate.

Definition 8 Suppose $\alpha>_{1} \beta$. We say that a particular occurrence of a subterm $\sigma_{\alpha}$ of $\alpha$ is unbroken in the reduction $\alpha>_{1} \beta$ iff either $\sigma_{\alpha}$ is not a subterm of the reduced redex and the reduced redex is not a subterm of $\sigma_{\alpha}$ or $\sigma_{\alpha}$ is a subterm of the reduced redex and it corresponds to an atomic subterm of the applied reduction axiom. A subterm $\sigma_{\alpha}$ is broken if a subterm (proper or not) of it is not unbroken. 
Example 7:

i. In $S(K S) x_{5} B>_{1} K S B\left(x_{5} B\right), K S$ is a subterm of the reduced redex and it corresponds to the variable $x_{1}$ of the reduction axiom $S x_{1} x_{2} x_{3}>_{1} x_{1} x_{3}\left(x_{2} x_{3}\right)$ we applied. Therefore $K S$ is unbroken.

ii. In $S(K S K K)>_{1} S(S K), K S$ is a subterm of the reduced redex and it does not correspond to an atomic subterm of the reduction axiom $K x_{1} x_{2}>x_{1}$ applied, because it corresponds to $K x_{1}$. Therefore $K S$ is broken. Also $K S K$, $K S K K$ and $S(K S K K)$ are broken because their subterm $K S$ is broken.

Definition 9 Let $\chi \equiv X \phi_{2} \ldots \phi_{m-1} \phi_{m} \phi_{m+1} \ldots \phi_{n}$ be an element of $\{X\}^{+}$ and $2 \leqslant m \leqslant n$. We say that $\phi_{m}$ is the rightmost deepest branch (RDB) of $\chi$ iff $\phi_{m}$ is a right applied subterm of $\chi$ containing an $X$ with c-number which is maximum in $\chi$ and $\phi_{m+1}, \ldots, \phi_{n}$ do not contain an $X$ with maximum c-number in $\chi$.

Example 8: Let us consider $X \phi_{2} \phi_{3} \phi_{4}$ where $\phi_{2} \equiv \phi_{4} \equiv X$ and $\phi_{3} \equiv X X X$. The maximum c-number is 3 and $\phi_{3}$ is the $\mathrm{RDB}$, because it contains an $X$ with c-number 3 and $\phi_{4}$ does not.

Lemma $8 \quad$ Let $\alpha \equiv \phi_{m} \psi_{m+1} \ldots \psi_{l}$ and $\beta \equiv X \phi_{2} \ldots \phi_{m-1} \phi_{m} \phi_{m+1} \ldots \phi_{n}$. Let $\phi_{i} \epsilon\{X\}^{+}$for $i=2, \ldots, n$ with $2 \leqslant m \leqslant n$ be in normal form. Let $\psi_{m+1}, \ldots, \psi_{l} \epsilon$ $\{X\}^{+}$with $m \leqslant l$ be in normal form. Let the maximum $c$-number in $\alpha$ and in $\beta$ be the same and let $\phi_{m}$ be the RDB of $\alpha$ and $\beta$. In these hypotheses: (i) $l=n$, and (ii) $\alpha \supsetneqq \beta$ (i.e., $\beta \notin R_{\alpha}$ ).

Proof: (i) $l=n$ because the maximum c-number in $\alpha$ and $\beta$ is the same. (ii) If a reduction is possible for $\alpha$ either $\phi_{m} \equiv X$ or $\phi_{m}$ is broken, because $\phi_{m+1}, \ldots, \psi_{l}$ are in normal form. If $\phi_{m} \equiv X$ the copies of $X$ with c-number equal to the maximum c-number in $\beta$ are 0 (because they are just 1 in $\alpha$ ) and obviously in that case $\alpha \ngtr \beta$. If $\phi_{m}$ in $\alpha$ is broken we have that:

1. $\phi_{m}$ in $\beta$ cannot derive from a subterm of $\psi_{m+1}$ or $\psi_{m+2}$ or . . or $\psi_{l}$ by Lemma 7 , because in $\phi_{m}$ in $\beta$ occurs an $X$ with maximum c-number and no $X$ with maximum c-number occurs in $\psi_{m+1}, \ldots \psi_{l}$ and NA property holds

2. $\phi_{m}$ in $\beta$ cannot derive from $\phi_{m}$ in $\alpha$ because $\phi_{m}$ in $\alpha$ is broken and $X$ does not have compositive effect

3. $\phi_{m}$ in $\beta$ cannot be obtained by composition of terms derived from subterms of $\psi_{m+1}, \ldots, \psi_{l}$ or proper subterms of $\phi_{m}$ in $\alpha$ because $X$ does not have compositive effect.

From 1, 2, and $3, \alpha \neq \beta$ follows.

Definition $10 \quad$ Among the right applied subterms of a term $\alpha \equiv \alpha_{1} \alpha_{2} \ldots \alpha_{n}$ where $\alpha_{1}$ is an atomic subterm, we define

1. the relation to be at the right hand side of (ROF) as follows:

$$
\forall i, j, 1 \leqslant i, j \leqslant n, \alpha_{i} \operatorname{ROF} \alpha_{j} \text { iff } i>j
$$

2. the relation to be at the left hand side of (LOF) as follows:

$$
\forall i, j 1 \leqslant i, j \leqslant n, \alpha_{i} \text { LOF } \alpha_{j} \text { iff } i<j
$$


Remark 5: In the case that $\alpha \equiv \alpha_{1} \ldots \alpha_{n}$ and all $\alpha_{i}$ 's are atomic, if $\alpha_{i}$ LOF $\alpha_{j}$ then $\alpha_{i}$ in $\operatorname{mark}(\alpha)$ has c-number greater than that of $\alpha_{j}$ in $\operatorname{mark}(\alpha)$. Analogously if $\alpha_{i}$ ROF $\alpha_{j}$ then $\alpha_{i}$ in $\operatorname{mark}(\alpha)$ has a c-number less than that of $\alpha_{j}$ in $\operatorname{mark}(\alpha)$.

Remark 6: If $\alpha_{i}$ ROF $\alpha_{j}$ then $\alpha_{j}$ LOF $\alpha$. If $\alpha_{i}$ ROF $\alpha_{j}$ then $\forall \gamma$ subterms of $\alpha_{i}, \forall \delta$ subterm of $\alpha_{j}, \gamma$ ROF $\delta$. Analogously using LOF instead of ROF and ROF instead of LOF.

Lemma 9 Consider $\chi_{1} \equiv X \phi_{2}^{(1)} \ldots \phi_{m}^{(1)} \ldots \phi_{n}^{(1)} \epsilon\{X\}^{+}$with $2 \leqslant m \leqslant n$ and $\chi_{2} \equiv X \phi_{2}^{(2)} \ldots \phi_{k}^{(2)} \ldots \phi_{t}^{(2)} \in\{X\}^{+}$with $2 \leqslant k \leqslant t$. Suppose:

1. $\chi_{1}>_{1} \chi_{2}$

2. maximum c-number in $\chi_{1}=$ maximum c-number in $\chi_{2}$

3. $\phi_{m}^{(1)} \equiv \phi_{k}^{(2)}$ and $\phi_{m}^{(1)}$ produces $\phi_{k}^{(2)}$

4. $\phi_{m}^{(1)}$ and $\phi_{k}^{(2)}$ contain an $X$ with maximum c-number in $\chi_{1}$ and $\chi_{2}$ respectively.

Under these hypotheses:

(i) $n-m=t-k$

(ii) no $\phi_{p}^{(1)}$ with $m<p \leqslant n$ produces any $\phi_{q}^{(2)}$ with $2 \leqslant q \leqslant k$

(iii) each $\phi_{p}^{(1)}$ with $m<p \leqslant n$ can only produce elements of the set $\left\{\phi_{i}^{(2)} \mid k<\right.$ $i \leqslant t\}$.

Proof: (i) Immediate.

(ii) Since $\phi_{m}^{(1)}$ produces $\phi_{k}^{(2)}$ and $\chi_{1}>_{1} \chi_{2}$ by applying the axiom $X x_{1} \ldots x_{m}>$ $\beta$, if $\phi_{m}^{(1)}$ corresponds to the variable $x_{i}$ in $X x_{1} \ldots x_{m}$, then $\phi_{k}^{(2)}$ corresponds to another occurrence of the same variable $x_{i}$ in $\beta$. Those two occurrences of the variable $x_{i}$ have the same c-number in $X x_{1} \ldots x_{m}$ and $\beta$ respectively, because otherwise $\phi_{m}^{(1)}$ and $\phi_{k}^{(2)}$ could not have a copy of $X$ with maximum c-number in $\chi_{1}$ and $\chi_{2}$, respectively. If a $\phi_{p}^{(1)}$ with $m<p \leqslant n$ produces a $\phi_{q}^{(2)}$ for any $q=2, \ldots, k$, that fact would imply that an $x_{j}$, with $j>i$ in $X x_{1} \ldots x_{m}$ such that $x_{j}$ ROF $x_{i}$, where $x_{i}$ in $X x_{1} \ldots x_{m}$ corresponds to $\phi_{m}^{(1)}$, would have been the same as an $x_{k}$ in $\beta$ such that $x_{k}$ LOF $x_{i}$, where $x_{i}$ in $\beta$ corresponds to $\phi_{k}^{(2)}$.

Since the two occurrences of $x_{i}$, above mentioned, have the same cnumber in $X x_{1} \ldots x_{m}$ and $\beta$, respectively, $X$ could not have NA property by Remark 1.

(iii) All $\phi_{p}^{(1)}$ 's with $m<p \leqslant n$ are unbroken because $\chi_{1}>_{1} \chi_{2}$ is a leftmost outermost reduction and $\phi_{m}^{(1)}$ is unbroken. From point ii and hypothesis 3 , point iii follows.

Lemma 10 Let $\phi_{i} \in\{X\}^{+}$for $i=2, \ldots, n$ with $n \geqslant 2$ be in normal form. Let $\chi_{0}$ be $X \phi_{2} \ldots \phi_{m-1} \phi_{m} \phi_{m+1} \ldots \phi_{n}$ where $2 \leqslant m \leqslant n$ and $\phi_{m}$ is the RDB of $\chi_{0}$. If $\chi_{0}>_{1} \chi_{1}>_{1} \ldots>_{1} \chi_{i}>_{1} \ldots>_{1} \chi_{k} \equiv \chi_{0}$ for $k \geqslant 1$ then

(i) $\phi_{m}$ which is the $R D B$ of $\chi_{k}$ derives from $\phi_{m}$ which is the $R D B$ of $\chi_{0}$

(ii) $\forall i, 0 \leqslant i \leqslant k$, a copy of $\phi_{m}$ occurs in $\chi_{i}$ and it is the $R D B$ of $\chi_{i}$ and $\phi_{m}$ which is the $R D B$ in $\chi_{i}$ produces the $\phi_{m}$ which is the $R D B$ of $\chi_{i+1}$ 
(iii) $\forall i, 0 \leqslant i \leqslant k-1, \phi_{m}$ which is the $R D B$ of $\chi_{i}$ is unbroken in the reduction $\chi_{i}>_{1} \chi_{i+1}$.

Proof: (i) $\phi_{m}$, which is the RDB of $\chi_{k}$, cannot be obtained as composition of subterms of $\phi_{2}, \ldots, \phi_{n}$ because $X$ does not have compositive effect; it cannot derive from a subterm of $\phi_{m+1}$ or ... or $\phi_{n}$ because in $\phi_{m}$ occurs an $X$ with maximum c-number and no $X$ with maximum c-number occurs in $\phi_{m+1}$ or $\ldots$ or $\phi_{n}$ (by Lemmas 5 and 7); and it cannot derive from a subterm of $\phi_{2}, \ldots, \phi_{m-1}$ because such a subterm could not have an $X$ with maximum c-number in $\chi_{k}$, say $d$, unless it had in $\chi_{0}$ (which is identical to $\chi_{k}$ ) an $X$ with a c-number strictly greater, than $d$ (by Lemma 5). Therefore the copy of $\phi_{m}$ which is RDB of $\chi_{k}$ derives from the copy of $\phi_{m}$ which is RDB in $\chi_{0}$.

(ii) The derivation of $\phi_{m}$ which is the RDB of $\chi_{k}$ from $\phi_{m}$ which is the RDB of $\chi_{0}$, since $X$ does not have compositive effect, is obtained by a sequence of $\phi_{m}$ 's such that $\forall i, 0 \leqslant i \leqslant k-1$, a copy of $\phi_{m}$ in $\chi_{i+1}$ derives from a copy of $\phi_{m}$ in $\chi_{i}$ and each copy is a right applied subterm of the corresponding combinator. In order to prove point ii we have to show that each $\phi_{m}$ of the abovementioned sequence of $\phi_{m}$ 's is the RDB of the corresponding $\chi_{i}$. Since we know (by point i) that $\phi_{m}$ in $\chi_{k}$ is the RDB of $\chi_{k}$, we have only to prove that: $\forall i, 1 \leqslant i \leqslant k$, if $\phi_{m}$ which is the RDB of $\chi_{i}$ derives from a copy of $\phi_{m}$ in $\chi_{i-1}$ then that copy of $\phi_{m}$ in $\chi_{i-1}$ is the RDB of $\chi_{i-1}$. In fact:

(1) an $X$ with maximum c-number must occur in that copy of $\phi_{m}$ in $\chi_{i-1}$ because otherwise that copy of $\phi_{m}$ would not produce a $\phi_{m}$ which is the RDB of $\chi_{i}$ (by Lemma 4); and

(2) no other right applied subterms of $\chi_{i-1}$ containing an $X$ with maximum c-number occur in $\chi_{i-1}$ on the right hand side of the copy of $\phi_{m}$ which produces the RDB of $\chi_{i}$, because if any would occur, it would produce in $\chi_{i}$ subterms containing copies of $X$ without maximum c-number by Lemma 9 (point iii) and therefore, by Lemma $6, \chi_{0}$ could not cycle.

(iii) If $\phi_{m}$, which is the RDB of $\chi_{i}$ for some $i$ such that $0 \leqslant i \leqslant k-1$, is broken in the reduction $\chi_{i}>_{1} \chi_{i+1}$ then $\chi_{i} \equiv \phi_{m} \psi_{m+1} \ldots \psi_{l}$ by Lemma 3 and no $k$ exists such that $\chi_{i}>_{k} \chi_{0}$ by Lemma 8 .

Lemma 11 Let $\phi_{i} \in\{X\}^{+}$for $i=2, \ldots, n$ with $n \geqslant 2$ be in normal form. Let $\chi_{0}$ be $X \phi_{2} \ldots \phi_{m-1} \phi_{m} \phi_{m+1} \ldots \phi_{n}$ where $m \leqslant n$ and $\phi_{m}$ is the RDB of $\chi_{0}$. Let $\chi_{0}>_{1} \chi_{1}>_{1} \ldots>_{1} \chi_{i}>_{1} \ldots>_{1} \chi_{k} \equiv \chi_{0}$ for $k \geqslant 1$. On these hypotheses:

(i) $\psi_{m}, \psi_{m+1}, \ldots, \psi_{n} \in\{X\}^{+}$exist such that $\chi^{\prime} \equiv X \phi_{2} \ldots \phi_{m-1} \psi_{m} \psi_{m+1} \ldots \psi_{n}$ cycles, and

(ii) $\psi_{m}, \psi_{m+1}, \ldots, \psi_{n}$ do not have $X$ 's with maximum c-number in $\chi^{\prime}$.

(Note that $\chi$ differs from $\chi^{\prime}$ also for the subterm $\phi_{m}$ which is transformed into $\left.\psi_{m}\right)$.

Proof: (i) Let us denote by $\phi_{j}^{(i)}$ for $2 \leqslant j \leqslant n$ the right applied subterms of $\chi_{i}$ for $i=0,1, \ldots, k$. By Lemma $10 \phi_{m}^{(k)}$ derives from $\phi_{m}^{(0)}$. Now let us prove the following:

Assertion $1 \quad$ Each $\phi_{m+1}^{(k)}, \ldots, \phi_{n}^{(k)}$ may be derived either from $\phi_{m}^{(0)}$ or $\phi_{m+1}^{(0)}$ or . . or $\phi_{n}^{(0)}$ as a copy of one of them or from a subterm of $\phi_{2}^{(0)}$ or . . or $\phi_{m-1}^{(0)}$. 
Proof of Assertion 1: Each $\phi_{j}^{(k)}$ with $m+1 \leqslant j \leqslant n$ :

1. cannot derive from a proper subterm of $\phi_{m+1}^{(0)}$ or . . or $\phi_{n}^{(0)}$ because during the reduction from $\chi_{0}$ to $\chi_{k} \equiv \chi_{0}, \phi_{m}$ is unbroken (by Lemma 10) and since we make only leftmost outermost reductions, also $\phi_{m+1}^{(0)}, \ldots, \phi_{n}^{(0)}$ are unbroken

2. cannot derive from a proper subterm of $\phi_{m}^{(0)}$ because it would imply that $\exists i, 0 \leqslant i \leqslant k-1$, such that the copy of $\phi_{m}$, which is the RDB of $\chi_{i}$ and derives from $\phi_{m}^{(0)}$ by Lemma 10 , is broken. In fact in each $\chi_{i}, 0 \leqslant i \leqslant k$, no other copies of $\phi_{m}$ are on the left hand side of the copy which is the RDB of $\chi_{i}$, because otherwise the copy of $\phi_{m}$ which is the RDB of $\chi_{i}$ could not contain a copy of $X$ with maximum c-number in $\chi_{i}$. Therefore since all reductions from $\chi_{0}$ to $\chi_{k}$ are head reductions, subterms of $\phi_{m}^{(0)}$ can only be derived by breaking in $\chi_{i}$, for some $i$ such that $0 \leqslant i \leqslant k-1$, a copy of $\phi_{m}$ which is the RDB of $\chi_{i}$. But in that case, by Lemma 10 , point iii, $\chi_{0}$ could not cycle.

3. cannot be obtained by composition of subterms because $X$ does not have compositive effect.

From 1, 2, and 3, Assertion 1 follows.

To continue the proof of Lemma 11(i), now let us choose:

1. $\psi_{m} \equiv X$

2. $\forall i, j, m+1 \leqslant i \leqslant n$ and $m \leqslant j \leqslant n$, if $\phi_{i}^{(k)}$ derives from $\phi_{j}^{(0)}$ (as a copy of it) then $\psi_{i} \equiv \psi_{j} \equiv X$

3. $\forall i, m+1 \leqslant i \leqslant n$, if $\phi_{i}^{(k)}$ derives from a subterm of $\phi_{2}^{(0)}$ or $\ldots$ or $\phi_{m-1}^{(0)}$ then $\psi_{i} \equiv \phi_{i}$.

With the above choices $\chi^{\prime} \equiv X \phi_{2} \ldots \phi_{m-1} \psi_{m} \psi_{m+1} \ldots \psi_{n}$ cycles because $\chi_{0}$ cycles and the subterm structure of $\chi^{\prime}$ preserves the same derivation relationships among subterms as they are in $\chi_{0}$.

(ii) Obviously $\psi_{m}, \psi_{m+1}, \ldots, \psi_{n}$ do not have $X$ 's with maximum c-number in $\chi^{\prime}$.

Lemma 12 No combinator $\bar{\chi} \equiv X \phi_{2} \ldots \phi_{n}$ exists in $\{X\}^{+}$such that $\phi_{2}, \ldots, \phi_{n}$ are in normal form and $\bar{\chi}$ does not have normal form.

Proof: Since by Lemma $1 R_{\bar{\chi}}$ is a finite set, we have to prove that $\bar{\chi}$ cannot cycle. The proof is by structural induction on $\bar{\chi}$.

Basis: $\bar{\chi} \equiv X$ does not cycle.

Induction step: $\bar{\chi} \equiv \phi_{1} \phi_{2}$. Suppose $\phi_{1}$ and $\phi_{2}$ are in normal form.

By iteratively applying Lemma 11 , if $\left(\phi_{1} \phi_{2}\right)$ cycles then also so does $\chi \equiv$ $X \psi_{2} \ldots \psi_{k}$ where $k \geqslant 2$ and $\forall i, 2 \leqslant i \leqslant k, \dot{\psi}_{i}$ is in normal form and the leftmost $X$ in $\chi$ has maximum c-number in $\chi$. But $\chi$ cannot cycle by Lemma 6 because at the first leftmost outermost reduction (if a reduction is possible at all) an $X$ with maximum c-number is deleted. Therefore $\phi_{1} \phi_{2}$ cannot cycle.

Proof of Theorem 1: Immediate from Lemmas 2 and 12. 
Example 9: Given $\beta=\{X\}$ such that $X x_{1} x_{2} x_{3} x_{4} x_{5}>x_{1} x_{2} x_{2} x_{4} x_{4} \forall \chi \in\{X\}^{+} \chi$ has normal form. In fact, $X$ has NA property and it does not have compositive effect.

Remark 7: If in Definition 1 we had $p>q$ instead of $p \geqslant q$ then the proof of Theorem 1 would have been immediate, using a "well order" argument (cf. [3]). In fact, at each contraction step the c-number of each copy of $X$ diminishes.

4 Some consequences of the basic theorem Given a combinator $\chi$, in general more than one redex occurs in it and therefore more than one combinator $\chi^{\prime}$ exists such that $\chi>_{1} \chi^{\prime}$.

Definition 11 A reduction strategy is a rule which chooses the redex (or the redexes) to be reduced in a term with more than one redex. when a reduction step must be performed.

Theorem 2 Given a proper combinator $X$ with $N A$ property and without compositive effect, $\forall \chi \in\{X\}^{+}$every reduction strategy applied to $\chi$ leads to its normal form.

Proof: By Theorem 1, $\forall \chi \in\{X\}^{+}$all subterms of $\chi$, which are themselves elements of $\{X\}^{+}$, have normal form.

Theorem 2 can also be extended to subbases with more than one basic combinator.

Theorem 3 Given a subbase $\mathcal{B}=\left\{X_{1}, \ldots, X_{n}\right\}$ such that $\forall X_{i} \in \mathcal{B} X_{i}$ has $N A$ property and doesn't have compositive effect, $\forall \chi \in \mathcal{B}^{+}$, every reduction strategy applied to $\chi$ leads to its normal form.

Proof: In the proofs of lemmas necessary for proving Theorem 1 we use only the facts that NA property holds and combinators don't have compositive effect.

Example 10: Given $\mathcal{B}=\left\{X_{1}, X_{2}\right\}$ where $X_{1} x_{1} x_{2} x_{3}>x_{1} x_{2} x_{2}$ and $X_{2} x_{1} x_{2} x_{3} x_{4}>$ $x_{1} x_{2} x_{2} x_{3}, \forall \chi \in \beta^{+} \chi$ has normal form. In fact, $X_{1}$ and $X_{2}$ have NA property and no compositive effect. For instance:

$$
\begin{aligned}
\chi \equiv X_{1}\left(X_{1} X_{2}\right)\left(X_{1} X_{1} X_{2}\right) X_{2} X_{2} X_{1} & { }_{1} X_{1} X_{2}\left(X_{1} X_{1} X_{2}\right)\left(X_{1} X_{1} X_{2}\right) X_{2} X_{1} \\
& { }_{1} X_{2}\left(X_{1} X_{1} X_{2}\right)\left(X_{1} X_{1} X_{2}\right) X_{2} X_{1} \\
& { }_{1} X_{1} X_{1} X_{2}\left(X_{1} X_{1} X_{2}\right)\left(X_{1} X_{1} X_{2}\right) X_{2} \\
& >_{1} X_{1} X_{2} X_{2}\left(X_{1} X_{1} X_{2}\right) X_{2} \\
& >_{1} X_{2} X_{2} X_{2} X_{2} .
\end{aligned}
$$

Theorem 3 improves the result in [2], page 180, where termination of a combinator $\chi \in \mathcal{B}^{+}$is guaranteed if there are no basic combinators in $\mathcal{B}$ with duplicative effect. According to Curry and Feys [2] the results about termination are as follows:

1. If all combinators in $\mathcal{B}$ are without duplicative effect then $\forall \chi \in \mathcal{B}^{+} \chi$ has normal form.

2. If some combinators in $\mathcal{B}$ have duplicative effect then it could be the case that: 
i. $\forall \chi \in \mathcal{B}^{+}, \chi$ has normal form (see Example 11.1)

ii. some $\chi$ 's in $\beta^{+}$do not have normal form (see Example 11.2).

Example 11:

1. Let us consider $\mathcal{B}=\{X\}$ such that $X x_{1} x_{2}>x_{1} x_{1}$.

All $\chi \in \mathcal{B}^{+}$have normal form because eventually the leftmost outermost redex will be of the form $X X \phi$ and from that reduction onward the number of right applied subterms of the subterm where the reduction occurred diminishes.

2. Let us consider $B=\{W\}$ such that $W x_{1} x_{2}>x_{1} x_{2} x_{2}$.

$W W W$ has no normal form because it cycles.

As a consequence of our result Case 2 is split into two subcases:

2.1 If all combinators in $\mathcal{B}$ have NA property and no compositive effect then $\forall \chi \in \mathcal{B}^{+} \chi$ has normal form.

2.2 If some combinators in $\mathcal{B}$ do not have NA property or they have compositive effect then it could be the case that:

i. $\forall \chi \in \mathcal{B}^{+} \chi$ has normal form (see Example 12.1)

ii. some $\chi$ 's in $\mathcal{B}^{+}$do not have normal form (see Example 12.2).

Example 12:

1. Let us consider any $\mathcal{B}=\{X\}$ where $X$ doesn't have NA property and has no duplicative effect (e.g., $X \equiv C$ where $C x_{1} x_{2} x_{3}>x_{1} x_{3} x_{2}$ ). By Curry's result, any $\chi \in\{X\}^{+}$has normal form.

2. We consider three cases:

$2.1 \mathcal{B}=\{S\}$ where $S$ does not have NA property and has compositive effect. If we denote $S S S$ by 3 then $S 33(S 33)$ does not have normal form. In fact, $3 x_{1} x_{2} \geqslant x_{1} x_{2}\left(S x_{1} x_{2}\right)$ and $s_{0} \equiv S 33(S 33) \geqslant s_{1} \equiv S 33(3(S 33)) \ldots \geqslant$ $s_{2} \equiv S 33(3(3(S 33)))$ and so on.

$2.2 \beta=\{W\}$ where $W$ does not have NA property and no compositive effect. $W W W$ doesn't have normal form.

2.3 $\mathcal{B}=\{X\}$ where $X$ is any combinator which has NA property and compositive effect.

In general, the problem of determining whether or not termination is guaranteed for all $\chi \in\{X\}^{+}$when NA property is the only condition on $X$ is open. In some particular cases that problem has a simple solution. For example, if $X x_{1} x_{2} x_{3}>x_{1}\left(x_{2} x_{2}\right)$, then termination is guaranteed for all $\chi \in\{X\}^{+}$(this is a consequence of Example 10).

5 Conclusions and related work We introduced the NA property for proving termination of combinators in Weak Combinatory Logic (WCL). The result is applicable also to term-rewriting systems.

The proof of the main theorem is based on the finiteness of the set of terms that can be obtained by reduction from a given combinator and on the absence of cycling reductions (cf. [6]). 
Some other methods have been used by other authors for similar proofs and we would like to refer to [3] and [4], in which "well ordering" and "value preserving" techniques were used for proving termination of "context free" tree rewriting systems. With regard to those methods we notice that: (i) NA property does not allow the direct application of a "well-ordering" technique because it defines a nonstrict order between the structure of the redex and the structure of the contractum; (ii) our termination result is obtained in WCL whose power of computation is the same as a Turing machine (i.e., a "type 0" grammar and not a "context free" one). We would like also to relate our study with Sanchis' result on termination of terms of typed $\lambda$-calculus [8]. Obviously WCL terms can be translated into $\lambda$-calculus, but, in the case of combinators with NA property, self application is possible and, in general, typed $\lambda$-calculus would not be sufficient for such a translation. Therefore our result is outside the scope of Sanchis'.

In general, for type 0 grammars the termination problem is undecidable, and our study is an effort in the direction of defining properties for extending the class of terms in which termination is decidable. It could also have useful applications in equational logic and in the theory of recursive equations, LISP and Lucid (as shown in [5]) as well as in type-free languages for tree manipulation (cf. [7]).

\section{REFERENCES}

[1] Abdali, K. S., "A lambda-calculus model of programming languages, Parts I and II," Journal of Computer Languages, vol. 1. (1976), pp. 287-301 and pp. 303-320.

[2] Curry, H. B. and R. Feys, Combinatory Logic, Vol. I, North-Holland, Amsterdam, 1958.

[3] Manna, Z. and S. Ness, "On termination of Markov algorithms," pp. $789-792$ in Proceedings of the Third Hawaii International Conference on System Science, ed. B. Granborg, 1970.

[4] Lipton, R. J. and L. Snyder, "On the halting of tree replacement systems," pp. 43-46 in Proceedings of the Conference on Theoretical Computer Science, ed. E. Ashcroft, University of Waterloo, Ontario, Canada, 1977.

[5] O’Donnell, M., "Subtree replacement systems: A unifying theory for recursive equations, LISP, lucid and combinatory logic," pp. 295-305 in ACM Theory of Computing, ed., J. Hopcroft, 1977.

[6] Pettorossi, A., "Sulla terminazione in classi subricorsive di algoritmi" ("On termination of subrecursive classes of algorithms"), pp. 405-412 in Proceedings of the AICA Congress, Genova, 1975.

[7] Pettorossi, A., "On type-free languages for tree manipulation inside weak combinatory logic," pp. 213-223 in Proceedings of the 2nd Congress of Lille; Les arbres en Algèbre et en Programmation, ed., G. Jacob, 1977.

[8] Sanchis, L., Course Notes in Lattice Semantics, Syracuse University (1978).

Centro di Studio dei Sistemi di

Controllo e Calcolo Automatici del C.N.R.

Via Eudossiana 18

00184 Roma, Italy

\section{Present address: IASI-CNR}

Via Buonarroti 12

00185 Roma, Italy 